\title{
Evaluation of energy spectrum CT for the measurement of thyroid iodine content
}

\author{
Weiguang Shao ${ }^{\dagger}$, Jingang Liu $^{\dagger}$ and Dianmei Liu ${ }^{*}$
}

\begin{abstract}
Background: This study aims to provide a reference for the diagnosis of iodine deficiency disorder by evaluating the normal thyroid iodine content by energy spectrum computed tomography (CT) and calculating the iodine content ratio of thyroid to sternocleidomastoid.

Methods: The thyroid glands of 226 patients were scanned by energy spectrum $C T$, and the images were analyzed using the GSI Viewer software. Based on the imaging findings, the iodine levels of the thyroid lobes as well as the bilateral sternocleidomastoids were evaluated, and their iodine content ratios were calculated.

Results: No statistically significant difference was found in the thyroid iodine content between the left and right thyroid lobes $(p>0.05)$. However, there was a significant difference in the thyroid iodine content between men and women $(p<0.01)$. Additionally, the thyroid iodine content was found to decrease gradually with age. The iodine content ratio of thyroid to sternocleidomastoid was $96.6271 \pm 33.2442$.
\end{abstract}

Conclusion: Gemstone energy spectrum CT can be used for the measurement of thyroid iodine content in the human body. It can play a significant role in the diagnosis of iodine deficiency disorder.

Keywords: Energy spectrum CT imaging, Thyroid, lodine content

\section{Background}

Iodine, an essential trace element for the human body, is closely related to the thyroid and is essential for the synthesis of the thyroid hormones. Iodine deficiency or excess iodine can cause thyroid dysfunction [1, 2], leading to abnormal iodine content in the thyroid tissues, which results in disorders of the thyroid and other organs of the body [3-5]. The iodine intake levels of the thyroid and in vivo storage concentrations can be evaluated by measurement of the iodine content of the thyroid tissue. These values can be used to determine whether the thyroid dysfunction is caused by iodine deficiency or excess, which is clinically significant in the diagnosis of thyroid diseases [6]. Previously, the iodine content of the body was indirectly determined by the measurement of urine iodine levels $[7,8]$ and thyroid iodine absorption rates [9]. However, the iodine content of the thyroid glands cannot be measured by these methods. Thyroid iodine content can be determined by

\footnotetext{
* Correspondence: dianmeiliu@163.com

${ }^{\dagger}$ Equal contributors

Department of Imaging Center, the Affiliated Hospital of Weifang Medical University, Weifang 261031, China
}

the conversion of thyroid CT values; however, X-rays, which are used to achieve excitation in this process, have a limited spectral energy range, which inevitably leads to inaccuracies in the CT values, thus affecting the results of quantitative diagnosis [10, 11]. Gemstone energy spectrum CT is based on the differences in the X-ray attenuation coefficients of different materials. This technique can be used to obtain not only monoergic images, but also substance-separation images [12, 13]. Iodinebased substance separation images are very sensitive to iodine deposition and exhibit good resolution of tissues such as the thyroid gland; they can, therefore, be used for the quantification of the thyroid iodine content. In the present study, the possibility of energy spectrum CT iodine-based substance-separation imaging completely or partly replacing the previous methods for the measurement of thyroid iodine content was evaluated.

\section{Methods Subjects \\ Patients who underwent cervical and thyroid CT imaging between October 2010 and May 2011 were enrolled in this}


study. All of the patients had undergone energy spectrum CT imaging of the thyroid and sternocleidomastoid. None of the patients had received thyroid preparations, iodine products, or special foods such as laver, kelp, or seaweed. Patients with cysts, adenoma, calcifications, inflammation, and other disorders and/or dysfunctions of the thyroid were excluded. A total of 226 subjects between the ages of 18 and 77 years (mean age, $46 \pm 17$ years), including 119 male and 107 female patients were enrolled. This study was approved by the ethics committee of an affiliated hospital of the Weifang Medical College, and informed written consent had been obtained from all of the patients.

\section{Computed tomography scanning and post-processing}

Energy spectrum CT scanning was performed using the Discovery CT750 high definition (HD) scanner (GE Healthcare, Milwaukee, WI, USA), with the following scanning parameters: section thickness, $5.0 \mathrm{~mm}$, with 5.0-mm intervals; thread interval, $0.984: 1$; speed, $39.37 \mathrm{~mm} /$ rotation; and rotation time, $0.8 \mathrm{~s}$. The imaging data were reconstructed at a reconstruction thickness and interval of $0.625 \mathrm{~mm}$ and transmitted to AW4.4 workstations, where they were analyzed using the GSI viewer software. Monoergic images with optimal thyroid contrast-to-noise ratios (CNRs) were obtained from the energy spectrum curve. On the iodine-based substance-separation images, the largest thyroid layer was selected for the measurement of iodine content in the right and left thyroid lobes within $50 \mathrm{~mm}^{2}$ circular areas. The iodine content was measured at two or three points on the upper pole of each lobe, and the mean value of these measurements was calculated. The iodine content in the bilateral sternocleidomastoids was also measured, and the iodine content ratio of thyroid to sternocleidomastoid was determined.

\section{Statistical analysis}

The data were recorded as the mean values \pm standard deviations (SD) and analyzed using the SSPS v13.0 (Chicago IL, USA) software. Comparison of the thyroid iodine content between the male and female patients was performed using the $t$-test, while comparison of the thyroid iodine content among different age groups was performed by analysis of variance (F-test). Pairwise comparisons between the groups were performed using the SNK q-test. The level of significance of the test was determined at $\alpha=0.05$, and statistical significance was determined at $p<0.05$.

\section{Results}

Thyroid iodine content in the right and left lobes

A total of 226 right and 225 left thyroid lobes (because of one patient with an absent left lobe) were evaluated.
The mean iodine content in the left lobe was $1.5230 \pm$ $0.4271 \mathrm{mg} / \mathrm{cm}^{3}$ and that in the right lobe was $1.5236 \pm$ $0.4365 \mathrm{mg} / \mathrm{cm}^{3}$ (Table 1). The difference in iodine content between the right and left lobes was not statistically significant $(t=0.0084 ; p>0.05)$.

\section{Thyroid iodine content in male and female patients and iodine content ratio of thyroid to sternocleidomastoid}

The mean value of the total iodine content in the thyroid glands was $1.5233 \pm 0.4318 \mathrm{mg} / \mathrm{cm}^{3}$. The difference in iodine content between the male and female patients was statistically significant $(t=3.4743 ; p<0.01 ;$ Fig. 1 and Table 2). The iodine content ratio of thyroid to sternocleidomastoid $\left(0.0161 \pm 0.0615 \mathrm{mg} / \mathrm{cm}^{3}\right)$ was $96.6271 \pm$ 33.2442 , and it showed no statistically significant differences between the male and female patients $(t=0.3817$; $p>0.3817$; Fig. 1 and Table 2).

\section{Optimal CNR of the thyroid glands}

Monoergic images with optimal CNR of the thyroid glands against the sternocleidomastoid were achieved at $57.0167 \pm 2.7647 \mathrm{keV}$ (Fig. 2).

\section{Thyroid iodine content in different age groups}

The thyroid iodine content showed significant differences among the different age groups evaluated in the present study $(F=9.66 ; p<0.01)$. Patients below 40 years of age had markedly higher thyroid iodine content than patients between the ages of 40 and 60 years and those above 60 years of age $(q=5.6195$ and 5.6195, respectively; $p<0.01$, both; Table 3). However, the difference in thyroid iodine content between patients between the ages of 40 and 60 years and those above 60 years of age was not statistically significant $(q=0.3166 ; p>0.3166$; Table 3).

\section{Discussion}

Characteristics of energy spectrum CT

Energy spectrum imaging was first studied in the 1970s [14]. This was followed by the clinical application of this research in dual energy imaging [15]. In the 2000s, it was almost possible to achieve substance separation with the dual-energy subtraction technique by means of CT scanning [16]. Using the instantaneous double $\mathrm{kVp}$ technique, it was possible to acquire a series of monoergic images at specific energy levels by means of energy spectrum $\mathrm{CT}$ in order to effectively avoid the beam

Table 1 Comparisons of the thyroid iodine content between the right and left lobes

\begin{tabular}{llcrl}
\hline & No. of cases & lodine content $(\mathrm{mg} / \mathrm{cc})$ & $t$ value & $P$ value \\
\hline Left lobe & 225 & $1.5230 \pm 0.4271$ & 0.0084 & 0.9933 \\
Right lobe & 226 & $1.5236 \pm 0.4365$ & & \\
\hline
\end{tabular}

Data were shown as mean $\pm S D$ 


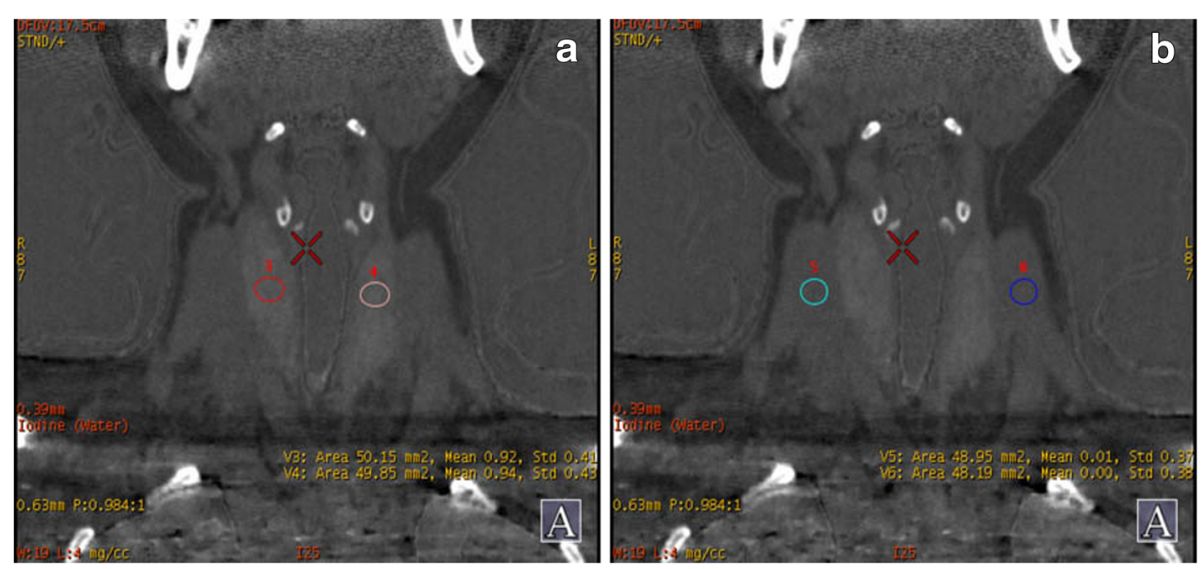

Fig. 1 a Was iodine-based image for determining the iodine content in thyroid glands. b was iodine-based image for determining the iodine content in bilateral sternocleidomastoids

hardening effect and improve the CNR of the images, thus obtaining stable and precise $\mathrm{CT}$ values. These advances resulted in the replacement of conventional CT, which is based on the variation of a single parameter, as the diagnostic modality of choice with energy and multiparameter-based CT evaluation [17]. Image acquisition by $\mathrm{CT}$ is based on the attenuation of X-rays in the objects of interest. Each material has its own characteristic curve correlating the changes of the mass absorption coefficient with energy. Because of the inherent differences in their energy attenuation coefficients, different materials in an object can be identified and quantified by excitation at two different energy levels. This is the physical basis of substance-separation imaging [18]. By means of substance-separation imaging, each structure can be broken down into separate substances with different X-ray attenuation coefficients, following which, contrast images of these substances can be acquired and used for the qualitative analysis of the contents. Since iodine-based substance-separation imaging exhibits high sensitivity in the imaging of iodine deposits, it aids in better visualization of iodine-rich tissues such as the thyroid glands. Therefore, iodine-based thyroid density images can be used for the quantitative analysis of the iodine content of the thyroid glands.

\section{Limitations of the previous evaluation methods}

Thyroid iodine content accounts for about $20-50 \%$ of the total iodine content of the human body. It has been reported that excessive iodine intake as well as its deficiency can lead to thyroid dysfunction, which exhibits a U-shaped relationship with iodine intake $[19,20]$. Measurement of iodine content can reveal the amount of iodine reserve in vivo as well as the recent iodine intake levels. Previous determination methods mostly focused on the measurement of either urine iodine $[7,8]$ or the rate of thyroid iodine absorption [9]. However, the results obtained by such methods can be affected by the iodine content contributed by food and renal and gastrointestinal functions. The iodine content of the thyroid can also be analyzed by measurement of the $\mathrm{CT}$ values using a specific formula; however, the spectral range of X-rays is limited. Moreover, conventional CT image acquisition cannot effectively avoid the beam hardening effect in order to obtain stable and accurate $\mathrm{CT}$ values; this leads to inaccuracies in quantitative analysis $[10,11]$.

\section{Advantages and clinical value of energy spectrum $\mathrm{CT}$ for the measurement of thyroid iodine content}

Gemstone energy spectrum CT can be used to effectively determine the iodine content of the thyroid using iodine-based substance-separation images transformed from the X-ray attenuation curve. Errors due to inaccuracies of the CT values can be avoided, and accurate measurements of the iodine levels in the body can be obtained. Based on the results of specimen imaging studies by Li et al. [21], dual-energy CT can be considered

Table 2 lodine content in thyroid and sternocleidomastoid of men and women

\begin{tabular}{|c|c|c|c|c|}
\hline \multirow[t]{2}{*}{ Groups } & \multirow[t]{2}{*}{ No. of cases } & \multicolumn{2}{|c|}{ lodine content $\left(\mathrm{mg} / \mathrm{cm}^{3}\right)$} & \multirow{2}{*}{$\begin{array}{l}\text { lodine ratio of thyroid to } \\
\text { sternocleidomastoid }\end{array}$} \\
\hline & & Thyroid glands & $\overline{\text { Sternocleidomastoid }}$ & \\
\hline Men & 119 & $1.6395 \pm 0.4105^{*}$ & $0.0175 \pm 0.0635$ & $94.6250 \pm 37.3621$ \\
\hline Women & 107 & $1.4238 \pm 0.3832$ & $0.0145 \pm 0.0613$ & $98.0000 \pm 29.0737$ \\
\hline
\end{tabular}

Data were shown as mean $\pm \mathrm{SD} .{ }^{*} P<0.01$, compared with women 

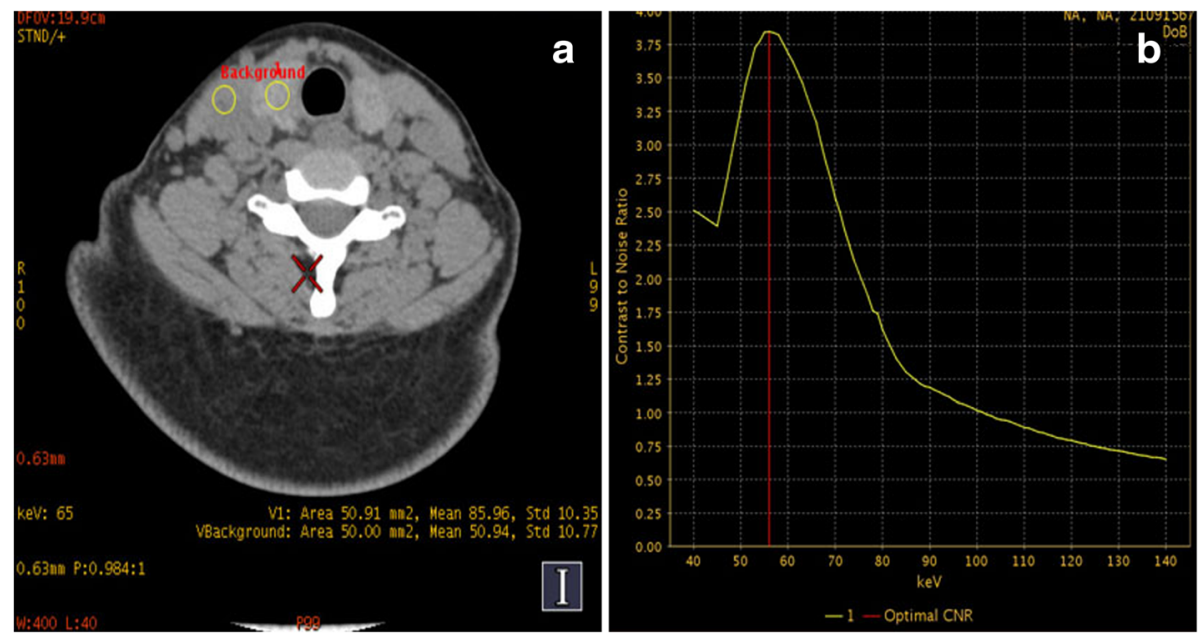

Fig. 2 a. Two ROls were drawn in right thyroid gland and ipsilateral sternocleidomastoid respectively. b. Using the ipsilateral sternocleidomastoid as a contrast, the right thyroid gland had the best contrast to noise ratio (57 kev) to the surrounding tissues

as a promising quantitative approach for the differentiation of malignant and benign thyroid nodules. In the present study, the results of analysis of normal thyroid glands revealed no statistically significant differences in iodine content between the left and right thyroid lobes; however, significant differences in thyroid iodine content were observed between the male and female subjects, which might be associated with the differences in endocrine hormonal levels between the two sexes. We also found a gradual decline in the thyroid iodine content with increasing age, which suggests that thyroid function, including iodine reserve and uptake, exhibits a tendency to decline with age. The results of comparison among the different age groups revealed no significant differences in thyroid iodine content between patients between the ages of 40 and 60 years and those above 60 years of age. However, the thyroid iodine content of the patients of both groups was lower compared to that of the patients below 40 years of age, which indicates that thyroid function might start to decline from the age of 40 . However, the relationship between age and thyroid function needs verification by further studies with larger sample sizes.

The iodine content ratio of thyroid to other tissues, which is normally about 100:1, is an important index in the diagnosis of iodine deficiency disorders; this ratio has been reported to be high as 400:1 in patients with iodine deficiency disorders [5]. In cases where the ratio is higher than 100:1, the concentration of iodine absorbed should be evaluated. In cases where the ratio is close to 400:1, the patients are considered as exhibiting serious iodine deficiency. In the present study, the iodine content ratio of thyroid to sternocleidomastoid was $96.6271 \pm 33.2442$. Therefore, this value can be used as the reference for the diagnosis of iodine deficiency disorders by measurement of the thyroid iodine content by energy spectrum CT. Gemstone energy spectrum CT can be used not only to accurately measure the iodine content of the thyroid glands and determine the iodine content ratio of thyroid to sternocleidomastoid, but also to determine the volume of the thyroid glands and evaluate any complications because of other thyroid diseases. Thus, energy spectrum CT is more advantageous than conventional $\mathrm{CT}$ in the evaluation of thyroid function and morphology.

\section{Limitations of this study}

First, the sample size in the present study was too small, which resulted in the inclusion of subjects of a wide range of ages. Moreover, because of the small sample size, the age groups could not be evaluated in terms of sex-specific differences in thyroid iodine content. Further studies with larger sample sizes including higher numbers of subjects of each age group are required for obtaining more reliable results. Second, we positioned the ROIs only in the upper poles of the thyroid glands; however, whether the distribution of iodine is consistent across the entire thyroid gland

Table 3 Comparisons of thyroid iodine content among different age groups

\begin{tabular}{|c|c|c|c|c|c|}
\hline Age groups & No. of cases & lodine content $\left(\mathrm{mg} / \mathrm{cm}^{3}\right)$ & & $q$ value & $P$ value \\
\hline$<40$ years $(\mathrm{a})$ & 59 & $1.7256 \pm 0.4631$ & a vs. b & 5.6195 & $<0.01$ \\
\hline 40 to 60 years $(b)$ & 96 & $1.4517 \pm 0.3643$ & a vs. C & 5.4158 & $<0.01$ \\
\hline$>60$ years $(c)$ & 71 & $1.4368 \pm 0.3465$ & b vs. c & 0.3166 & $>0.05$ \\
\hline
\end{tabular}


has yet to be confirmed. Positioning of the ROIs only in the upper or lower poles of the thyroid gland might lead to inconsistency in the results. Third, the equipment used was expensive and involved the use of radioactive imaging agents. Therefore, its application in the routine screening of abnormal thyroid function might be challenging.

\section{Conclusion}

Gemstone energy spectrum CT can be used for the evaluation of thyroid iodine content in the human body. In the present study, imaging by this method revealed a gradual decrease in thyroid iodine content with age. Therefore, gemstone energy spectrum $\mathrm{CT}$ is a promising tool for the diagnosis of iodine deficiency disorder.

\section{Abbreviations}

$C T$, computed tomography; $H D$, high definition; $C N R$, contrast to noise ratio; $\mathrm{SD}$, standard deviation.

\section{Authors' contributions}

DL designed and performed the study. She also reviewed and edited the manuscript; WS and JL acquired and analyzed the data and wrote the paper; all authors read and approved the final manuscript.

\section{Competing interests}

The authors declare that they have no competing interests.

\section{Ethics approval and consent to participate}

This study was approved by the ethics committee of an affiliated hospital of the Weifang Medical College, and informed written consent had been obtained from all of the patients.

\section{Received: 17 February 2015 Accepted: 4 August 2016}

Published online: 12 August 2016

\section{References}

1. Tan L, Sang Z, Shen J, Liu H, Chen W, Zhao N, et al. Prevalence of thyroid dysfunction with adequate and excessive iodine intake in Hebei Province, People's Republic of China. Public Health Nutr. 2014;17:1-6.

2. Yan YR, Liu Y, Huang H, Lv QG, Gao XL, Jiang J, et al. lodine nutrition and thyroid diseases in Chengdu, China: an epidemiological study. QJM. 2014 [Epub ahead of print]

3. Sun X, Shan Z, Teng W. Effects of increased iodine intake on thyroid disorders. Endocrinol Metab (Seoul). 2014:29:240-7.

4. Hetzel BS. The development of a global program for the elimination of brain damage due to iodine deficiency. Asia Pac J Clin Nutr. 2012;21:164-70.

5. Chen ZP, Hetzel BS. Cretinism revisited. Best Pract Res Clin Endocrinol Metab. 2010;24:39-50.

6. Bürgi H. lodine excess. J Best Pract Res Clin Endocrinol Metab. 2010;24:107-15.

7. World Health Organinzation, United Nations Children's Fund, International Council for Control of lodine Deficiency Disorders. Assessment of iodine deficiency disorders and monitoring their elimination: a guide for programme managers. 3rd ed. Geneva: World Health Organinzation; 2007. p. 37-54.

8. Boasquevisque PC, Jarske RD, Dias CC, Quintaes IP, Santos MC, Musso C. Correlation between iodine urinary levels and pathological changes in thyroid glands. Arq Bras Endocrinol Metabol. 2013;57:727-32.

9. Narumi S, Nagasaki K, Ishii T, Muroya K, Asakura Y, Adachi M, et al. Nonclassic TSH resistance: TSHR mutation carriers with discrepantly high thyroidal iodine uptake. J Clin Endocrinol Metab. 2011;96:E1340-5.

10. Goodsitt MM, Christodoulou EG, Larson SC. Accuracies of the synthesized monochromatic $C T$ numbers and effective atomic numbers obtained with a rapid kVp switching dual energy CT scanner. Med Phys. 2011;38:2222-32.

11. Wang L, Liu B, Wu XW, Wang J, Zhou Y, Wang WQ, et al. Correlation between $\mathrm{CT}$ attenuation value and iodine concentration in vitro: discrepancy between gemstone spectral imaging on single-source dualenergy CT and traditional polychromatic X-ray imaging. J Med Imaging Radiat Oncol. 2012;56:379-83.
12. Duan X, Wang J, Yu L, Leng S, McCollough CH. CT scanner X-ray spectrum estimation from transmission measurements. Med Phys. 2011;38:993-7.

13. Ascenti G, Siragusa C, Racchiusa S, lelo I, Privitera G, Midili F, et al. Stone-targeted dual-energy $\mathrm{CT}$ : a new diagnostic approach to urinary calculosis. AJR Am J Roentgenol. 2010;195:953-8.

14. Chiro GD, Brooks RA, Kessler RM, Johnston GS, Jones AE, Herdt JR, et al. Tissue signatures with dual-energy computed tomography. Radiology. 1979;131:521-3.

15. Kalender WA, Klotz E, Suess C. Vertebral bone mineral analysis: an integrated approach with CT. Radiology. 1987;164:419-23.

16. Flohr TG, McCollough CH, Bruder H, Petersilka M, Gruber $\mathrm{K}$, Süss $\mathrm{C}$, et al. First performance evaluation of a dual-source CT (DSCT) system. Eur Radiol. 2006:16:256-68.

17. Zhang D, Li X, Liu B. Objective characterization of GE discovery CT750 HD scanner: gemstone spectral imaging mode. Med Phys. 2011:38:1178-88.

18. Anderson NG, Butler AP, Scott NJ, Cook NJ, Butzer JS, Schleich N, et al. Spectroscopic (multi-energy) CT distinguishes iodine and barium contrast material in MICE. Eur Radiol. 2010;20:2126-34.

19. Markou K, Georgopoulos N, Kyriazopoulou V, Vagenakis AG. lodine-induced hypothyroidism. Thyroid. 2001;11:501-10.

20. Roti E, Uberti ED. lodine excessive and hyperthyroidism. Thyroid. 2001:11:493-500

21. Li M, Zheng XP, Li JY, Yang YL, Lu C, Xu H, et al. Dual-energy computed tomography imaging of thyroid nodule specimens: comparison with pathologic findings. Invest Radiol. 2012;47(1):58-64.
Submit your next manuscript to BioMed Central and we will help you at every step:

- We accept pre-submission inquiries

- Our selector tool helps you to find the most relevant journal

- We provide round the clock customer support

- Convenient online submission

- Thorough peer review

- Inclusion in PubMed and all major indexing services

- Maximum visibility for your research

Submit your manuscript at www.biomedcentral.com/submit 